\title{
El papel de la Universidad Autónoma del Estado de México-campus El Cerrillo como refugio de la diversidad de aves en el Valle de Toluca, México
}

Ramírez-Albores, Jorge E. y Pérez Suárez, Marlín

El papel de la Universidad Autónoma del Estado de México-campus El Cerrillo como refugio de la diversidad de aves en el Valle de Toluca, México

CIENCIA ergo-sum, vol. 25, núm. 3, noviembre 2018-febrero 2019 | e32

Universidad Autónoma del Estado de México, México

Esta obra está bajo una Licencia Creative Commons Atribución-NoComercial-SinDerivar 4.0 Internacional.

Ramírez-Albores, J. E. y Pérez Suárez, M. (2018). El papel de la Universidad Autónoma del Estado de México-campus El Cerrillo como refugio de la diversidad de aves en el Valle de Toluca, México. CIENCIA ergo-sum, 25(3). https://doi.org/10.30878/ces.v25n3a10 


\title{
El papel de la Universidad Autónoma del Estado de México-campus El Cerrillo como refugio de la diversidad de aves en el Valle de Toluca, México
}

The role of the Universidad Autonoma del Estado de Mexico-El Cerrillo campus as a shelter for bird diversity in the Toluca Valley, Mexico

Jorge E. Ramirez-Albores

Universidad Nacional Autónoma de México, México

jorgeramirez22@hotmail.com

Recepción: 07 de febrero de 2017 Aprobación: 05 de septiembre de 2017

\author{
Marlin Pérez Suárez \\ Universidad Autónoma del Estado de México, México \\ mperezs@uaemex.mx
}

\section{Resumen:}

Se da a conocer la diversidad de aves presente en la Universidad Autónoma del Estado de México-campus El Cerrillo en Toluca de Lerdo, Estado de México. El inventario avifaunístico se realizó a través de recorridos con puntos de conteo dentro del campus y alrededores. Se registraron un total de 141 especies de aves, la mayor riqueza de especies fue terrestre (107) y el resto fue de hábitos acuáticos y subacuáticos (34). Del total de especies, cuatro están enlistadas bajo algún estatus de riesgo en la NOM-059Semarnat-2010. La diversidad de aves registrada sugiere que esta área representa una fuente importante de recursos alimenticios, espacio de anidación para especies residentes, así como sitio de paso para especies migratorias.

Palabras Clave: avifauna, campus universitario, fragmentación, humedal, Bordo Las Maravillas.

\section{Abstract:}

We present the bird diversity of the Universidad Autonoma del Estado de Mexico-El Cerrillo campus in Toluca de Lerdo, Mexico State. Bird inventory was realized through transects with point counts inside the campus and surroundings. A total of 141 bird species were recorded, the greatest species richness were terrestrial birds (107), and the rest were species with aquatic and semiaquatic habits (34). Of the total species, four are listed under some risk status in NOM-059-Semarnat-2010. Bird diversity recorded on campus suggests that this area represents an important source of food resources, as nesting area by resident species and well as passage site by migratory species.

KEYWORDS: birds, university campus, fragmentation, wetland, board Las Maravillas.

\section{INTRODUCCIÓN}

La pérdida y fragmentación del hábitat original transforman el paisaje y son amenazas significativas para la biodiversidad (Fahrig, 2003). Estos procesos pueden modificar la diversidad y composición de la biota nativa, y con ello la intensidad de las interacciones ecológicas (Murcia, 1995). Las aves son un grupo ecológica y taxonómicamente muy diversificado, de distribución mundial y con una marcada sensibilidad a los cambios ambientales (Morrison, 1986). Ante esta situación, uno se pregunta cómo responderán las aves si se tiene en cuenta que es un grupo vulnerable ante los cambios en las características de su hábitat, en especial a los relacionados con la estructura de la vegetación. A pesar del gran impacto causado por los distintos usos de suelo en los ecosistemas naturales, muchas especies de aves utilizan los nuevos ambientes generados por el hombre al hacer uso de su plasticidad ecológica y conductual (Wong y Candolin, 2014). Sin embargo, varios estudios han demostrado que dicha transformación del hábitat original hacia asentamientos humanos, pastizales o zonas agrícolas las ha afectado negativamente, ya que se ha modificado su diversidad, composición y también se ha reducido su tamaño poblacional (Laurance y Bierregaard, 1997; Renjifo, 1999; Daily et al., 2001). Por tanto, ante la constante y progresiva transformación de los ambientes naturales, las áreas verdes dentro y adyacentes a los recintos urbanos o matrices con diferentes usos de suelo están destinadas a 
convertirse en refugios y hábitats alternativos para muchas especies de aves silvestres (Sulaiman et al., 2013; Tryjanowski et al., 2017) y pueden actuar como corredores biológicos si presentan conectividad, sitios de descanso durante la migración de algunas especies o fuente temporal de recursos alimenticios. Por estas razones, el estudio de las comunidades de aves en estos sitios puede aportar información relevante sobre el estado de conservación del hábitat, la presencia/ausencia de algunas especies indicadoras de la calidad de éste, su abundancia y tiempo de residencia (Morrison, 1986; Wong y Candolin, 2014).

La ubicación del Estado de México en la parte central del país, aunada a su historia geológica, relieve, topografía, gran variedad de climas y tipos de vegetación heterogéneos, le confiere cualidades para contener una amplia biodiversidad (López et al., 2009). Esto sugiere que es una entidad en la que existe variedad considerable de aves residentes, la cual aumenta por ser una ruta de migración de muchas otras. De acuerdo con De Sucre et al. (2009), la diversidad de aves en el Estado de México es de 490 especies, que constituye 46.4\% respecto al país (AOU, 2017). A pesar de esta variedad, los estudios aún son escasos, pero se concentran principalmente en áreas boscosas como el Nevado de Toluca y Sierra de Nanchititla (UrbinaTorres et al., 2012; Sánchez-Jasso et al., 2013), en grandes cuerpos de agua como las ciénegas de Lerma y Texcoco (González et al., 1999; Manterola, 1999) y en áreas verdes dentro de la Zona Metropolitana de la Ciudad de México (Duarte, 2001; Acuña, 2014), siendo casi nulo el conocimiento en ambientes urbanos o en otros puntos de la entidad. En México, en los últimos años se han realizado algunos estudios sobre la comunidad de aves en ambientes urbanos y suburbanos (Ramírez-Albores, 2008; Carbo-Ramírez et al., 2011). Estos estudios se han concentrado en la parte central del país, así como otros pocos en otras regiones, los cuales hacen mención sobre la importancia de estos sitios como zonas de refugio, anidación y de alimentación para muchas especies de aves (tabla 1). En la ciudad de Toluca y zona conurbada, pese a su urbanización, existen áreas con cobertura arbórea que pueden ofrecer un lugar alterno para las aves, como las zonas arboladas de Ciudad Universitaria de la Universidad Autónoma del Estado de México, Parque Metropolitano Bicentenario, Cerro La Teresona, Cerro del Espíritu Santo o cuerpos de agua a los alrededores de la ciudad. Sin embargo, no hay estudios realizados de la diversidad avifaunística que albergan estos sitios. Por este motivo, el objetivo del artículo es dar a conocer la diversidad de aves presente en la Universidad Autónoma del Estado de México-campus El Cerrillo, el cual está ubicado a 13 kilómetros de la ciudad de Toluca, Estado de México.

TABLA 1

Riqueza de aves registrada en centros universitarios en ambientes urbanos del país

\begin{tabular}{|c|c|c|c|c|}
\hline Sitio & $\begin{array}{c}\text { Riqueza } \\
\text { de especies } \\
\text { de aves }\end{array}$ & $\begin{array}{l}\text { Distancia a la zona } \\
\text { urbana o poblado } \\
\text { más cercano }(\mathrm{km})\end{array}$ & $\begin{array}{l}\text { Distancia a zona con } \\
\text { vegetación natural } \\
\text { más cercana }(\mathrm{km})\end{array}$ & Referencia \\
\hline $\begin{array}{l}\text { Centro Universitario, Universidad Autónoma de } \\
\text { Tamaulipas (Ciudad Victoria, Tamaulipas) }\end{array}$ & 114 & $\begin{array}{l}\text { Dentro de la zona } \\
\text { periurbana }\end{array}$ & 1.5 & $\begin{array}{l}\text { Gómez-Moreno et } \\
\text { al. (2016) }\end{array}$ \\
\hline $\begin{array}{l}\text { Facultad de Estudios Superiores Iztacala, } \\
\text { UNAM (Los Reyes Iztacala, Estado de México) }\end{array}$ & 100 & $\begin{array}{l}\text { Dentro de la zona } \\
\text { urbana }\end{array}$ & 7.0 & $\begin{array}{l}\text { Duarte (2001), } \\
\text { Acuña (2014) }\end{array}$ \\
\hline $\begin{array}{l}\text { Centro Universitario en Ciencias Bilógicas y } \\
\text { Agropecuarias, Universidad de Guadalajara } \\
\text { (Zapopan, Jalisco) }\end{array}$ & 70 & $\begin{array}{l}\text { Dentro de la zona } \\
\text { periurbana }\end{array}$ & 2.0 & $\begin{array}{l}\text { MacGregor-Fors } \\
(2005)\end{array}$ \\
\hline $\begin{array}{l}\text { Ciudad Universitaria, Universidad Autónoma } \\
\text { del Estado de Hidalgo (Pachuca, Hidalgo) }\end{array}$ & 36 & $\begin{array}{l}\text { Dentro de la zona } \\
\text { periurbana }\end{array}$ & 7.5 & $\begin{array}{l}\text { Carbo-Ramírez et } \\
\text { al. (2011) }\end{array}$ \\
\hline $\begin{array}{l}\text { Centro de Enseñanza, Investigación y Extensión } \\
\text { en Producción Agro-Silvo-Pastoril de la } \\
\text { Facultad de Medicina Veterinaria y Zootecnia- } \\
\text { UNAM (Chapa de Mota, Estado de México) }\end{array}$ & 68 & 0.5 & 1.4 & Calvo (2015) \\
\hline $\begin{array}{l}\text { Facultad de Estudios Superiores Zaragoza } \\
\text { campus II-UNAM (Ciudad de México) }\end{array}$ & 70 & $\begin{array}{l}\text { Dentro de la zona } \\
\text { urbana }\end{array}$ & 25.0 & $\begin{array}{l}\text { Ramírez-Albores } \\
(2008)\end{array}$ \\
\hline
\end{tabular}




\section{LAS AVES DEL CAMPUS}

El Cerrillo es un campus universitario que pertenece a la Universidad Autónoma del Estado de México y está localizado al noreste del municipio de Toluca aproximadamente a $13 \mathrm{~km}$ del centro de Toluca. El campus se ubica en las coordenadas $19^{\circ} 24^{\prime}$ de latitud norte y $99^{\circ} 41^{\prime}$ de longitud oeste, a una altitud de $2610 \mathrm{msnm}$, cuenta con una superficie de 49.6 ha y alberga diferentes facultades e institutos como la Facultad de Ciencias, Facultad de Química, Facultad de Ciencias Agrícolas, Facultad de Medicina Veterinaria y Zootecnia y el Instituto de Ciencias Agrícolas y Rurales. Este espacio universitario se encuentra en las afueras de la localidad de San Cayetano de Morelos, inmerso dentro de una matriz de uso agrícola con asentamientos humanos a los alrededores y árboles dispersos. En el campus pueden encontrarse árboles de eucalipto (Eucalyptus spp.), pino (Pinus spp.), encino (Quercus spp.), casuarina (Casuarina equisetifolia), fresno (Fraxinus spp.), ciprés (Cupressus spp.), durazno (Prunus persica), entre otros, así como campos de cultivo experimental de maíz, jitomate, frijol, girasol y una pequeña área con cactáceas y yucas. Muy cerca se encuentra el humedal Bordo Las Maravillas: un cuerpo de agua con una profundidad entre 60 y $80 \mathrm{~cm}$ y una superficie de $20 \mathrm{ha}$, en donde crecen poblaciones de especies vegetales higrófilas como juncos (Typha latifolia, Schoenoplectus lacustris) y en una superficie mayor se encuentra bordeado por árboles de sauce llorón (Salix babylonica) y de ciprés (Cupressus spp.). A los alrededores del campus y de este humedal se pueden encontrar otros cuerpos de agua temporal y permanente con diferentes superficies, arbolado disperso y líneas arboladas utilizadas como barreras rompevientos.

A la fecha se han registrado un total de 141 especies de aves pertenecientes a 15 órdenes y 43 familias (Anexo). Estos registros constituyen 28.7\% de la avifauna reportada para el Estado de México y 13.3\% respecto al país (De Sucre et al., 2009; AOU, 2017). Las familias con más especies son Parulidae con 13 especies y Anatidae con 12 especies, seguidas de Passerellidae con 11, Tyrannidae con 10 y Ardeidae con siete. Estas familias en conjunto incluyeron $40 \%$ del total de especies registradas. Referente a la estacionalidad 76 especies son residentes (53.9\%), 47 visitantes de invierno (33.3\%), 11 transitorias (7.8\%), una visitante de verano (0.7\%) y seis exóticas (4.2\%) (Anexo). La mayor riqueza de especies fueron aves terrestres (107 e s pecies) y el resto fueron especies con hábitos acuáticos y subacuáticos (34 especies) (figura 1). En cuanto a 1 estatus de conservación, cuatro especies se encuentran enlistadas bajo protección especial (Pr) en la NOM-059-Semarnat-2010 (Semarnat, 2010): Gavilán de Cooper, Aguililla Rojinegra, Aguililla de Swainson y Halcón Peregrino, además de registrar la presencia de dos especies endémicas de México (González-García y Gómez de Silva, 2003): Chipe Rojo y Rascador Corona Negra. 


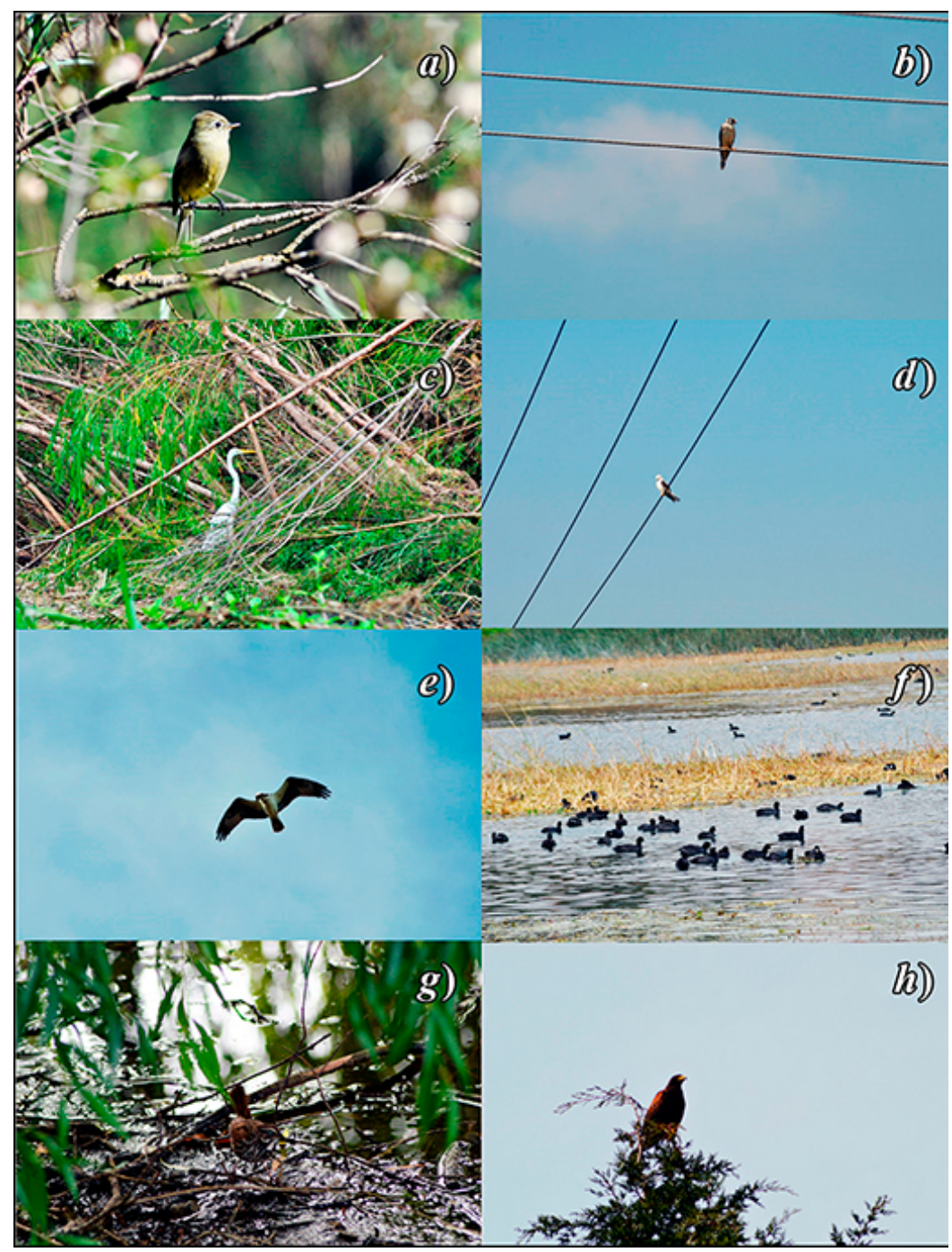

FIGURA 1

Aves registradas en el campus El Cerrillo

Nota: a) Reyezuelo Matraquita (Regulus calendula), b) Cernícalo Americano (Falco sparverius), c) Garza Blanca (Ardea alba), d) Milano Cola Blanca (Elanus leucurus), e) Águila Pescadora (Pandion haliaetus), $f$ ) Parvada de Gallareta Americana (Fulica americana),

g) Gorrión Cantor (Melospiza melodia), h) Aguililla Rojinegra (Parabuteo unicinctus) Fuente: fotos de J. E. Ramírez-Albores.

En el campus se pueden observar individuos de especies acuáticas y subacuáticas como patos, garzas e ibis, así como playeros (Anexo), los cuales están en constante desplazamiento dada la distribución de los cuerpos de agua dentro y a sus alrededores. Asimismo, suelen trasladarse a otros humedales o a la Presa Antonio Alzate (a $5.7 \mathrm{~km}$ del campus), Ciénegas de Lerma (a $26 \mathrm{~km}$ ) o Cieneguillas de Mañones (a $10.8 \mathrm{~km}$ ), por mencionar algunos. Entre los individuos observados, éstos pertenecen a las siguientes especies Cerceta Alas Verdes, Pato Mexicano, Garza Blanca, Garza Dedos Dorados, Garza Ganadera, Garza Nocturna Corona Negra, Ibis Ojos Rojos y Chorlo Tildío, que utilizan este humedal como sitio de descanso y alimentación. Además, se observan esporádicamente y en bajo número de individuos especies asociadas a áreas boscosas que utilizan el arbolado disperso o las líneas de arbolado como sitios de refugio o movimiento hacia áreas con mayor cobertura de bosque como el Carpintero de Pechera Común, Carpintero Bellotero, Bajapalos Pecho Blanco, Tepradorcito Americano y Pavito Alas Negras, consideradas indicadoras de la calidad del hábitat (Temple y Wiens, 1989; Canterbury et al., 2000), así como las asociadas a áreas abiertas: Gorrión Cola Blanca, Gorrión Sabanero, Tordo Sargento, Tordo Ojos Rojos, principalmente por la presencia de cultivos y zonas de pastoreo. También se observan parvadas mixtas de especies como Tordo Sargento, Tordo Cabeza Amarilla, Tordo Ojos Rojos 
y Tordo Cabeza Café, las cuales llegan a conformar grupos de más de 1000 individuos. La reducción de la cobertura forestal en fragmentos muy pequeños tiene consecuencias negativas para las comunidades de aves, pues se ve disminuida la diversidad considerablemente. Se presentan extinciones selectivas en fragmentos (Gimenes y Anjos, 2003) que tienden a reducir el número de especies especialistas y mantienen en su mayoría especies de hábitos generalistas. Por ejemplo, dado los usos de suelo a los alrededores del campus se encuentran especies de hábitos generalistas que son comunes como la Paloma Doméstica, Tortolita Cola Larga, Pinzón Mexicano, Gorrión Doméstico, Estornino Pinto y Zanate Mexicano (Crooks et al., 2004), las cuales son consideradas indicadoras de perturbación. Otro grupo importante son las especies considerada exóticas invasoras, es decir, aquellas que han sido introducidas a otra región diferente a la de su distribución natural y que se han establecido y colonizado en nuevos ambientes causando impactos a la biota nativa (Richardson $e t$ al., 2000) como la Paloma Doméstica, Garza Ganadera, Paloma de Collar Turca, Estornino Pinto y Gorrión Doméstico, originarias del Viejo Mundo, así como el Zanate Mexicano (originaria de la Costa del Golfo de México). Dos casos en particular que llaman la atención son el de la Cotorra Argentina y la Paloma de Collar Turca, pues han sido introducidas en varios países como ave ornamental. La Cotorra Argentina fue observada en áreas de cultivos a los alrededores del poblado de San Cayetano próximos al campus (Salgado-Miranda $e t$ al., 2016). Según Salgado-Miranda et al. (2016), los individuos de esta especie provienen de una población establecida en San Bartolomé Tlatelulco en Metepec (a $22 \mathrm{~km}$ dirección sur del campus). Sin embargo, a la fecha esta especie no ha sido avistada en el campus y tampoco en el sitio reportado. El segundo caso es el registro reciente dentro del campus de la Paloma de Collar Turca que realiza desplazamientos cortos como con actividad reproductiva (nidificando), además de observarla en áreas de cultivo cercanas, así como en el poblado de San Cayetano. Es probable que los individuos de esta especie hayan sido introducidos recientemente por los pobladores locales, ya que no había sido registrada en monitoreos anteriores.

\section{ANÁLISIS PROSPECTIVO Y CONCLUSIONES}

La importancia biológica que representan las aves va desde la polinización, dispersión de semillas, control poblacional, degradación de organismos muertos e indicadoras de la calidad ambiental hasta su apreciación estética y recreativa. Sin embargo, la transformación continua de los ambientes naturales en la entidad a causa de los asentamientos humanos, agricultura y ganadería abarcan en su conjunto más de $65 \%$ de la superficie de la entidad (López et al., 2009). Todo esto constituye un paisaje simplificado inmerso o adyacente a una matriz con diferentes usos de suelo, por lo que los espacios verdes y los relictos de vegetación natural en los alrededores de estas zonas transformadas se convierten en espacios urbanos que proporcionan un hábitat potencial para la permanencia de diversas especies faunísticas, en particular las aves, donde pueden encontrar sitios alternativos de alimentación, anidación y refugio.

El campus El Cerrillo y el Bordo Las Maravillas albergan una alta diversidad de aves en comparación con otros centros universitarios en ambientes urbanos o periurbanos del país (tabla 1). Lo anterior puede deberse a diversos atributos de las distintas áreas geográficas universitarias como $a$ ) superficie, $b$ ) tipo de vegetación, $c$ ) distancia respecto a los bordes de las ciudades y $d$ ) grado de urbanización, entre otros (Villard et al., 2000). La contribución de este artículo representa el conocimiento básico de la biodiversidad, el cual constituye información esencial para la toma de decisiones en la gestión ambiental y en la conservación de la biodiversidad. Las áreas verdes como el Campus El Cerrillo y el Bordo Las Maravillas proporcionan sitios de refugio, anidación, de paso y como corredor para muchas especies de aves de la región. Por ello es necesario realizar más investigación a corto y largo plazo que tienda a relacionar la estructura y funcionalidad de la comunidad de aves con las características de los hábitats, así como estudios poblacionales y aspectos ecológicos de algunas especies (con estatus de riesgo o endémicas), además de impulsar la conservación de áreas boscosas y los remanentes de vegetación, la creación de áreas verdes y el desarrollo de programas de educación ambiental que permitan conocer la diversidad. Con esto, la planificación del paisaje urbano 
podría potenciar la conectividad entre remanentes de vegetación natural y áreas verdes y así favorecer la fauna silvestre que se encuentra a los alrededores de las zonas urbanas y áreas de cultivo contribuyendo a la conservación de la biota nativa (Angold et al., 2006) y reducir la fragmentación y perturbación del ecosistema de la región.

\section{REFERENCIAS}

Acuña, J. D. (2014). Avifauna de la FES Iztacala, UNAM. Estudio comparativo (tesis de licenciatura). Estado de México: Facultad de Estudios Superiores Iztacala, UNAM.

Angold, P., Sadler, J., Hill, M., Pullin, A., Rushton, S., Austin, K., Small, E., Wood, B., Wadsworth, R., Sanderson, R. y Thompson, K. (2006). Biodiversity in urban habitat patches. Science of the Total Environment, 360(1-3), 196-204.

AOU (American Ornithologist's Union). (2017). Checklist of North and Middle American birds. Disponible en http://checklist.aou.org.

Berlanga, H., Gómez de Silva, H., Vargas-Canales, V. M., Rodríguez-Contreras, V., Sánchez-González, L. A., OrtegaÁlvarez, R. y Calderón-Parra, R. (2015). Aves de México: lista actualizada de especies y nombres comunes. México: Conabio.

Calvo, M. J. (2015). Avifauna registrada durante el periodo 2006-2011 en el Centro de Enseñanza, Investigación y Extensión en Producción Agro-Silvo-Pastoril (CEIEPASP), Chapa de Mota, Estado de México (tesis de licenciatura). México: Facultad de Medicina Veterinaria y Zootecnia, UNAM.

Canterbury, G. E., Martin, T. E., Petit, D. R., Petit, L. J. y Bradford, D. F. (2000). Bird community and habitat as ecological indicators of forest condition in regional monitoring. Conservation Biology, 14, 544-558.

Carbo-Ramírez, P., Zuria, I. y Romero-González, M. P. (2011). Riqueza, abundancia y dinámica espacio-temporal de la comunidad de aves de ciudad universitaria, Universidad Autónoma del Estado de Hidalgo, Pachuca, México. El Canto del Centzontle, 2, 29-47.

Crooks, K. R., Suárez, A. V. y Bolger, D. T. (2004). Avian assemblages along a gradient of urbanization in a highly fragmented landscape. Biological Conservation, 115, 451-462.

Daily, G. C., Ehrlich, P. R. y Sánchez-Azofeifa, G. A. (2001). Countryside biogeography: use of human-dominated habitats by the avifauna of southern Costa Rica. Ecological Applications, 11, 1-13.

De Sucre, A. E., Ramírez-Bastida, P., Gómez de Silva, H., Ramírez, S. (2009). Aves, en G. Ceballos, R. List, G. Garduño, R. López Cano, M. J. Muñozcano Quintanar, E. Collado y J. E. San Román (Comps.), La diversidad biológica del Estado de México. Estudio de Estado (pp.131-144). Toluca: Gobierno del Estado de México.

Duarte, M. T. (2001). Caracterización de la comunidad de aves de la UNAM campus Iztacala (tesis de licenciatura). Estado de México: Escuela Nacional de Estudios Profesionales Iztacala, UNAM.

Fahrig, L. (2003). Effects of habitat fragmentation on biodiversity. Annual Review of Ecology, Evolution, and Systematics, 34, 487-515.

Gimenes, M. R. y Anjos, L. (2003). Efeitos da fragmentação florestal sobre as comunidades de aves. Acta Scientarum Biological Sciences, 2, 391-402.

Gómez-Moreno, V.C., Niño-Maldonado, S. y Sánchez-Reyes, U.J. (2016). Lista ornitológica del Centro Universitario de Ciudad Victoria, Tamaulipas, México. Huitzil, 17, 33-43.

González, L. A., Ramírez, P., Varona, D. E., Cortés, E., Hernández, B., López, L. M., Márquez-Valdelamar, L. M., Alcántara, J. L., Ayala, D. E., Galindo, J. M., Néquiz, V. y Escalante, P. (1999). AICA 01-Lago de Texcoco. Disponible en http://conabio.gob.mx/aicas_progs/.

González-García, F. y Gómez de Silva, H. (2003). Especies endémicas: riqueza, patrones de distribución y retos para su conservación, en H. Gómez de Silva y A. Oliveras de Ita (Eds.), Conservación de aves. Experiencias en México (pp. 150-194). México: IPAMEX-CONABIO-National Fish and Wildlife Foundation. 
Howell, S. N. y Webb, S. (1995). A guide to the birds of Mexico and northern Central America. Oxford: Oxford University Press

Laurance, W. F., y Bierregaard, R. O. (1997). Tropical forest remnants: Ecology, management, and conservation of fragmented communities. Chicago: The University of Chicago Press.

López, R., Becerril, G., Benítez, C. y Cuevas, S. (2009). El medio físico, biológico y social, en G. Ceballos, R. List, G. Garduño, R. López Cano, M. J. Muñozcano Quintanar, E. Collado y J. E. San Román (Coords.), La diversidad biológica del Estado de México. Estudio de Estado (pp. 49-62). Toluca de Lerdo: Gobierno del Estado de México.

MacGregor-Fors, I. (2005). Listado ornitológico del Centro Universitario de Ciencias Biológicas y Agropecuarias, Universidad de Guadalajara, Jalisco, México: un espacio suburbano. Huitzil, 6,1-6.

Manterola, C. (1999). AICA 11-Ciénegas de Lerma. Disponible en http://conabio.gob.mx/aicas_progs/.

Morrison, M. L. (1986). Bird populations as indicators of environmental changes, en R. F. Johnston (Ed.), Current Ornithology (pp. 429-451). New York: Plenum Press.

Murcia, C. (1995). Edge effects in fragmented forests: implications for conservation. Trends in Ecology \& Evaluation, 2, 58-62.

Navarro-Sigüenza, A. G., Rebón-Gallardo, M. F., Gordillo-Martínez, A., Peterson, A.T., Berlanga, H. y SánchezGonzález, L.A. (2014). Biodiversidad de aves. Revista Mexicana de Biodiversidad, 85, 476-495.

Ramírez-Albores, J. E. (2008). Comunidad de aves de la Facultad de Estudios Superiores Zaragoza campus II, UNAM, Ciudad de México. Huitzil, 9, 12-19.

Renjifo, L. M. (1999). Composition change in subandean avifauna after long-term forest fragmentation. Conservation Biology, 13, 1124-1139.

Richardson, D. M., Pyšek, P., Rejmánek, M., Barbour, M. G., Panetta, F. D. y West, C. J. (2000). Naturalization and invasion of alien plants: concepts and definitions. Diversity and Distributions, 6, 93-107.

Salgado-Miranda, C., Medina, J. P., Sánchez-Jasso, J. M., y Soriano-Vargas, E. (2016). Registro altitudinal más alto en México para la cotorra argentina (Myiopsitta monachus). Huitzil, 17, 155-159.

Sánchez-Jasso, J. M., Aguilar-Miguel, X., Medina-Castro, J. P. y Sierra-Domínguez, G. (2013). Riqueza específica de vertebrados en un bosque reforestado del Parque Nacional Nevado de Toluca, México. Revista Mexicana de Biodiversidad, 84, 360-373.

Semarnat (Secretaría del Medio Ambiente y Recursos Naturales). (2010). Norma Oficial Mexicana NOM-059Semarnat-2010. Protección ambiental-especies nativas de México de flora y fauna silvestres-categorías de riesgo y especificaciones para su inclusión, exclusión o cambio-lista de especies en riesgo. México.

Sulaiman, S., Mohamad, N. H. e Idilfitri, S. (2013). Contribution of vegetation in urban parks as habitat for selective bird community. Procedia-Social and Behavioral Sciences, 85, 267-281.

Temple, S. A., y Wiens, J. A. (1989). Bird populations and environmental changes: can birds be bio-indicators? American Birds, 43, 260-270.

Tryjanowski, P., Morelli, F., Mikula, P., Krištín, A., Indykiewicz, P., Grzywaczewski, G., Kronenberg, J. y Jerzak, L. (2017). Bird diversity in urban green space: a large-scale analysis of differences between parks and cementeries in central Europe. Urban Forestry \& Urban Greening, 27, 264-271.

Urbina-Torres, F., Monroy-Vilchis, O., González-Martínez, A., Amador-Solís, C. L. R. y Celis-Murillo, A. (2012). Nuevos registros de aves para el Estado de México. Revista Mexicana de Biodiversidad, 83, 1241-1243.

Villard, M. A., Trzcinski y Merriam, G. (2000). Fragmentation effects on forest birds: relative influence of woodland cover and configuration on landscape occupancy. Conservation Biology, 13, 774-783.

Wong, B. B. M. y Candolin, U. (2014). Behavioral responses to changing environments. Behavioral Ecology, 26, 665-673. 
ANEXO

Lista de las aves registradas en el campus El Cerrillo de la Universidad Autónoma del Estado de México

\begin{tabular}{|c|c|c|c|}
\hline Orden / Familia & Especie & Nombre común & Estacionalidad \\
\hline \multirow{12}{*}{ Anseriformes / Anatidae } & Dendrocygna autumnalis & Pijije Alas Blancas & Transitorio \\
\hline & Dendrocygna bicolor & Pijije Canelo & Transitorio \\
\hline & Anas strepera & Pato Friso & Visitante de invierno \\
\hline & Anas americana & Pato Chalcuán & Visitante de invierno \\
\hline & Anas diazi & Pato Mexicano & Residente \\
\hline & Anas discors & Cerceta Alas Azules & Visitante de invierno \\
\hline & Anas cyanoptera & Cerceta Canela & Residente \\
\hline & Anas clypeata & Pato Cucharón Norteño & Visitante de invierno \\
\hline & Anas acuta & Pato Golondrino & Visitante de invierno \\
\hline & Anas crecca & Cerceta Alas Verdes & Visitante de invierno \\
\hline & Aythya affinis & Pato Boludo Menor & Visitante de invierno \\
\hline & Oxyura jamaicensis & Pato Tepalcate & Visitante de invierno \\
\hline Galliformes / Odontophoridae & Colinus virginianus & Codorniz Cotuí & Residente \\
\hline \multirow{3}{*}{ Podicipediformes / Podicipedidae } & Tachybaptus dominicus & Zambullidor menor & Transitorio \\
\hline & Podilymbus podiceps & Zambullidor Pico Grueso & Residente \\
\hline & Podiceps nigricollis & Zambullidor Orejón & Visitante de verano \\
\hline \multirow{5}{*}{ Columbiformes / Columbidae } & Columba livia & Paloma Doméstica & Exótica \\
\hline & Columbina inca & Tortolita Cola Larga & Residente \\
\hline & Streptopelia decaocto & Paloma de Collar Turca & Exótica \\
\hline & Zenaida asiatica & Paloma Alas Blancas & Residente \\
\hline & Zenaida macroura & Huilota Común & Residente \\
\hline Cuculiformes / Cuculidae & Geococcyx californianus & Correcaminos Norteño & Residente \\
\hline Caprimulgiformes / Caprimulgidae & Chordeiles acutipennis & Chotacabras Menor & Residente \\
\hline Apodiformes / Apodidae & Chaetura vauxi & Vencejo de Vaux & Transitorio \\
\hline \multirow{5}{*}{ Trochilidae } & Colibri thalassinus & Colibrí Orejas Violetas & Residente \\
\hline & Lampornis clemenciae & Colibrí Garganta Azul & Residente \\
\hline & Selasphorus platycercus & Zumbador Cola Ancha & Residente \\
\hline & Cynanthus latirostris & Colibrí Pico Ancho & Residente \\
\hline & Hylocharis leucotis & Zafiro Orejas Blancas & Residente \\
\hline \multirow{4}{*}{ Gruiformes / Rallidae } & Rallus limicola & Rascón Cara Gris & Residente \\
\hline & Porzana carolina & Polluela Sora & Visitante de invierno \\
\hline & Gallinula galeata & Gallineta Frente Roja & Residente \\
\hline & Fulica americana & Gallareta Americana & Residente \\
\hline Charadriiformes / Recurvirostridae & Himantopus mexicanus & Monjita Americana & Residente \\
\hline Charadriidae & Charadrius vociferus & Chorlo Tildío & Residente \\
\hline \multirow{6}{*}{ Scolopacidae } & Calidris bairdii & Playero de Baird & Transitorio \\
\hline & Limnodromus scolopaceus & Costurero Pico Largo & Visitante de invierno \\
\hline & Gallinago delicata & Agachona norteamericana & Visitante de invierno \\
\hline & Phalaropus tricolor & Falaropo pico largo & Transitorio \\
\hline & Actitis macularius & Playero Alzacolita & Visitante de invierno \\
\hline & Tring a flavipes & Patamarilla Menor & Visitante de invierno \\
\hline
\end{tabular}


Lista de las aves registradas en el campus El Cerrillo de la Universidad Autónoma del Estado de México (continúa)

\begin{tabular}{|c|c|c|c|}
\hline Orden / Familia & Especie & Nombre común & Estacionalidad \\
\hline \multirow{7}{*}{ Ciconiiformes / Ardeidae } & Ardea herodias & Garza Morena & Visitante de invierno \\
\hline & Ardea alba & Garza Blanca & Visitante de invierno \\
\hline & Egretta thula & Garza Dedos Dorados & Visitante de invierno \\
\hline & Bubulcus ibis & Garza Ganadera & Exótica \\
\hline & Butorides virescens & Garcita Verde & Residente \\
\hline & Nycticorax nycticorax & Garza Nocturna Corona Negra & Residente \\
\hline & Nyctinassa violacea & Garza Nocturna Corona Clara & Visitante de invierno \\
\hline Threskiornithidae & Plegadis chihi & Ibis Ojos Rojos & Residente \\
\hline \multirow{2}{*}{ Cathartiformes / Cathartidae } & Coragyps atratus & Zopilote Común & Residente \\
\hline & Cathartes aura & Zopilote Aura & Residente \\
\hline Accipitriformes / Pandionidae & Pandion haliaetus & Águila Pescadora & Transitorio \\
\hline \multirow{6}{*}{ Accipitridae } & Elanus leucurus & Milano Cola Blanca & Visitante de invierno \\
\hline & Circus cyaneus & Gavilán Rastrero & Visitante de invierno \\
\hline & Accipiter cooperi & Gavilán de Cooper & Visitante de invierno \\
\hline & Parabuteo unicinctus & Aguililla Rojinegra & Transitorio \\
\hline & Buteo swainsoni & Aguililla de Swainson & Transitorio \\
\hline & Buteo jamaicensis & Aguililla Cola Roja & Residente \\
\hline Strigiformes / Tytonidae & Tyto alba & Lechuza de Campanario & Residente \\
\hline Strigidae & Megascops kennicottii & Tecolote Occidental & Residente \\
\hline \multirow{3}{*}{ Piciformes / Picidae } & Melanerpes formicivorus & Carpintero Bellotero & Residente \\
\hline & Picoides scalaris & Carpintero Mexicano & Residente \\
\hline & Colaptes auratus & Carpintero de Pechera Común & Residente \\
\hline \multirow{3}{*}{ Falconiformes / Falconidae } & Falco sparverius & Cernícalo Americano & Visitante de invierno \\
\hline & Falco columbarius & Halcón Esmerejón & Visitante de invierno \\
\hline & Falco peregrinus & Halcón Peregrino & Residente \\
\hline \multirow{10}{*}{ Passeriformes / Tyrannidae } & Camptostoma imberbe & Mosquerito Chillón & Residente \\
\hline & Contopus cooperi & Papamoscas Boreal & Transitorio \\
\hline & Contopus pertinax & Papamoscas José María & Residente \\
\hline & Empidonax affinis & Papamoscas Pinero & Residente \\
\hline & Empidonax fulvifrons & Papamoscas Pecho Canela & Residente \\
\hline & Sayornis nigricans & Papamoscas Negro & Residente \\
\hline & Sayornis phoebe & Papamoscas Fibí & Visitante de invierno \\
\hline & Sayornis saya & Papamoscas Llanero & Visitante de invierno \\
\hline & Pyrocephalus rubinus & Papamoscas Cardenalito & Residente \\
\hline & Tyrannus vociferus & Tirano Chibiú & Residente \\
\hline Laniidae & Lanius ludovicianus & Verdugo Americano & Residente \\
\hline Vireonidae & Vireo huttoni & Vireo Reyezuelo & Residente \\
\hline Corvidae & Corvus corax & Cuervo Común & Residente \\
\hline Alaudidae & Eremophila alpestris & Alondra Cornuda & Residente \\
\hline \multirow{3}{*}{ Hirundinidae } & Tachycineta thalassina & Golondrina Verdemar & Visitante de invierno \\
\hline & Stelgidopteryx serripennis & Golondrina Alas Aserradas & Transitorio \\
\hline & Hirundo rustica & Golondrina Tijereta & Residente \\
\hline Aegithalidae & Psaltriparus minimus & Sastrecillo & Residente \\
\hline Sittidae & Sitta carolinensis & Bajapalos Pecho Blanco & Residente \\
\hline
\end{tabular}


Lista de las aves registradas en el campus El Cerrillo de la Universidad Autónoma del Estado de México (continúa)

\begin{tabular}{|c|c|c|c|}
\hline Orden / Familia & Especie & Nombre común & Estacionalidad \\
\hline Certhidae & Certhia americana & Trepadorcito Americano & Residente \\
\hline \multirow{4}{*}{ Troglodytidae } & Catherpes mexicanus & Saltapared Barranqueño & Residente \\
\hline & Troglodytes aedon & Saltapared Común & Visitante de invierno \\
\hline & Cistothorus palustris & Saltapared Pantanero & Visitante de invierno \\
\hline & Thryomanes bewickii & Saltapared Cola Larga & Residente \\
\hline Polioptilidae & Polioptila caerulea & Perlita Azulgris & Residente \\
\hline Regulidae & Regulus calendula & Reyezuelo Matraquita & Visitante de invierno \\
\hline \multirow{3}{*}{ Turdidae } & Sialia mexicana & Azulejo Garganta Azul & Residente \\
\hline & Catharus occidentalis & Zorzal Mexicano & Residente \\
\hline & Turdus migratorius & Mirlo Primavera & Residente \\
\hline \multirow{2}{*}{ Mimidae } & Toxostoma curvirostre & Cuicacoche Pico Curvo & Residente \\
\hline & Mimus polyglottos & Centzontle Norteño & Residente \\
\hline Sturnidae & Sturnus vulgaris & Estornino Pinto & Exótica \\
\hline Bombycillidae & Bombycilla cedrorum & Chinito & Visitante de invierno \\
\hline Ptiliogonatidae & Ptiliogonys cinereum & Capulinero Gris & Residente \\
\hline Passeridae & Passer domesticus & Gorrión Doméstico & Exótica \\
\hline \multirow{2}{*}{ Fringillidae } & Haemorhous mexicanus & Pinzón Mexicano & Residente \\
\hline & Spinus psaltria & Jilguerito Dominico & Residente \\
\hline \multirow{13}{*}{ Parulidae } & Parkesia noveboracensis & Chipe Charquero & Visitante de invierno \\
\hline & Mniotilta varia & Chipe Trepador & Visitante de invierno \\
\hline & Oreothlypis celata & Chipe Oliváceo & Visitante de invierno \\
\hline & Oreothlypis ruficapilla & Chipe Cabeza Gris & Visitante de invierno \\
\hline & Geothlypis tolmiei & Chipe Lores Negros & Visitante de invierno \\
\hline & Geothlypis trichas & Mascarita Común & Residente \\
\hline & Setophaga petechia & Chipe Amarillo & Visitante de invierno \\
\hline & Setophaga coronata & Chipe Rabadilla Amarilla & Visitante de invierno \\
\hline & Setophaga townsendi & Chipe de Townsend & Visitante de invierno \\
\hline & Setophaga occidentalis & Chipe Cabeza Amarilla & Visitante de invierno \\
\hline & Cardellina pusilla & Chipe Corona Negra & Visitante de invierno \\
\hline & Cardellina rubra & Chipe Rojo & Residente \\
\hline & Myioborus miniatus & Pavito Alas Negras & Residente \\
\hline \multirow{10}{*}{ Passerellidae } & Atlapetes pileatus & Rascador Gorra Negra & Residente \\
\hline & Aimophila rufescens & Zacatonero Canelo & Residente \\
\hline & Melozone fusca & Rascador Viejita & Residente \\
\hline & Spizella passerina & Gorrión Cejas Blancas & Residente \\
\hline & Spizella pallida & Gorrión Pálido & Visitante de invierno \\
\hline & Pooecetes gramineus & Gorrión Cola Blanca & Visitante de invierno \\
\hline & Chondestes grammacus & Gorrión Arlequín & Visitante de invierno \\
\hline & Passerculus sandwichensis & Gorrión Sabanero & Visitante de invierno \\
\hline & Melospiza melodia & Gorrión Cantor & Residente \\
\hline & Junco phaeonotus & Junco Ojos de Lumbre & Residente \\
\hline
\end{tabular}


Lista de las aves registradas en el campus El Cerrillo de la Universidad Autónoma del Estado de México (continúa)

\begin{tabular}{|llll}
\hline Orden / Familia & Especie & Nombre común & Estacionalidad \\
\hline \multirow{4}{*}{ Icteridae } & Xanthocephalus xanthocephalus & Tordo Cabeza Amarilla & Visitante de invierno \\
& Sturnella magna & Pradero Tortillaconchile & Residente \\
& Icterus abeillei & Calandria Flancos Negros & Residente \\
& Agelagius phoeniceus & Tordo Sargento & Residente \\
& Molothrus aeneus & Tordo Ojos Rojos & Residente \\
& Molothrus ater & Tordo Cabeza Café & Residente \\
& Quiscalus mexicanus & Zanate Mayor & Exótica \\
& Piranga rubra & Piranga Roja & Visitante de invierno \\
& Piranga ludoviciana & Piranga Capucha Roja & Visitante de invierno \\
& Pheucticus melanocephalus & Picogordo Tigrillo & Residente \\
Cardinalidae & Passerina caerulea & Picogordo Azul & Residente \\
& Passerina cyanea & Colorín Azul & Visitante de invierno \\
& Passerina versicolor & Colorín Morado & Residente \\
Thraupidae & Diglossa baritula & Picochueco Vientre Canela & Residente \\
\hline
\end{tabular}

Fuente: elaboración propia con base en Berlanga et al. (2015) y AOU (2017).

Nota: el orden taxonómico se basa en AOU (2017), los nombres comunes de acuerdo con Berlanga et al. (2015) y la estacionalidad de acuerdo con Howell y Webb (1995): a) residente (especie que se reproduce y permanece durante todo el año en el área de estudio), $b$ ) visitante de invierno (especie que se reproduce en latitudes más septentrionales y pasan el invierno en el área de estudio), c) transitorio (especie visitante no reproductor sólo presente durante la migración en primavera o verano) y $d$ ) se considera exótica a una especie introducida en una nueva área geográfica (Richardson et al., 2000). El estatus de riesgo se basa de acuerdo con la NOM-059-Semarnat-2010 (Semarnat, 2010).

\section{BY-NC-ND}

